\title{
Butyrate stimulates hepatic gluconeogenesis in mouse primary hepatocytes
}

\author{
XUEYING JI ${ }^{1 *}$, FEIYE ZHOU ${ }^{1 *}$, YUQING ZHANG ${ }^{1}$, RUYUAN DENG $^{1}$, WAN XU ${ }^{1}$, \\ MENGYAO BAI $^{1}$, YUN LIU ${ }^{1}$, LI SHAO ${ }^{2}$, XIAO WANG ${ }^{1}$ and LIBIN ZHOU $^{1}$ \\ ${ }^{1}$ Department of Endocrine and Metabolic Diseases, Shanghai Institute of Endocrine and Metabolic Diseases, \\ Shanghai Clinical Center for Endocrine and Metabolic Diseases, Ruijin Hospital, \\ Shanghai Jiaotong University School of Medicine, Shanghai 200025; ${ }^{2}$ The Very Important Person Department, \\ East Hospital, Shanghai Tongji University School of Medicine, Shanghai 200120, P.R. China
}

Received February 7, 2017; Accepted July 20, 2017

DOI: 10.3892/etm.2018.7136

\begin{abstract}
Butyrate is a major short-chain fatty acid (SCFA) produced by microbial fermentation of dietary fiber in the gastrointestinal tract. Butyrate is also a well-known broad-spectrum histone deacetylase (HDAC) inhibitor. Butyrate has been reported to improve energy metabolism in rodents, which is associated with its beneficial effects on skeletal muscle, brown fat tissue and pancreatic $\beta$-cells. The present study investigated the direct effect of butyrate on hepatic gluconeogenesis in mouse primary hepatocytes and the underlying mechanism. Isolated mouse primary hepatocytes were incubated with sodium butyrate, other HDAC inhibitors and other SCFAs. Hepatic glucose production was measured and gluconeogenic gene expression was detected by polymerase chain reaction analysis. The phosphorylation of cyclic adenosine monophosphate (cAMP) response element binding protein (CREB) was assessed by western blot analysis. The results revealed that sodium butyrate dose-dependently increased hepatic glucose production and gluconeogenic gene expression in isolated mouse primary hepatocytes.
\end{abstract}

Correspondence to: Professor Libin Zhou, Department of Endocrine and Metabolic Diseases, Shanghai Institute of Endocrine and Metabolic Diseases, Shanghai Clinical Center for Endocrine and Metabolic Diseases, Ruijin Hospital, Shanghai Jiaotong University School of Medicine, 197 Ruijin 2nd Road, Shanghai 200025, P.R. China

E-mail: libinzhou99@hotmail.com

Professor Li Shao, The Very Important Person Department, East Hospital, Shanghai Tongji University School of Medicine, 150 Jimo Road, Shanghai 200120, P.R. China

E-mail: dongfangshaoli@126.com

*Contributed equally

Key words: butyrate, gluconeogenesis, short chain fatty acid, histone deacetylase, cyclic adenosine monophosphate response element binding protein
Trichostatin A, a potent broad-spectrum HDAC inhibitor, had the opposite effect. Similar to sodium butyrate, propionate, which is another SCFA, promoted hepatic glucose production and gluconeogenic gene expression in the presence or absence of gluconeogenic substrates, which were further enhanced by cAMP. Furthermore, sodium butyrate also increased the accumulation of intracellular ATP and induced the phosphorylation of CREB in mouse hepatocytes. In conclusion, the present study suggested that butyrate stimulates hepatic gluconeogenesis and induces gluconeogenic gene expression as a substrate and cAMP/CREB signaling activator.

\section{Introduction}

Metabolic syndrome is a cluster of risk factors, including obesity, type 2 diabetes, dyslipidemia, hypertension and cardiovascular disease (1). Dietary intervention is a potential strategy to prevent and treat metabolic syndrome. It has been revealed that fiber-enriched diets improve obesity, insulin sensitivity and glucose tolerance, which is attributed to the production of short-chain fatty acid (SCFA) mediated by the gut microbiota (2-5). The most abundant SCFAs in the gut include acetate, propionate and butyrate (6). Although the major source of butyrate is the fermentation of dietary fibers, it is also contained in butter and cheese (7). Butyrate supplementation has been demonstrated to ameliorate diet-induced obesity, dyslipidemia, insulin resistance and glucose intolerance in mice (8). However, the mechanisms underlying the beneficial effect of butyrate has remained largely elusive.

It is well accepted that butyrate acts not only as a signaling molecule for the G-protein-coupled-receptor 41 (GPR41) and GPR43, but also as a wide-spectrum histone deacetylase (HDAC) inhibitor $(9,10)$. Histone deacetylases regulate gene transcription by deacetylation of proteins, including histone proteins and transcription factors. In recent years, HDAC has emerged as a novel molecular target in the treatment of type 2 diabetes. It has been demonstrated that HDACs are crucial regulators of pancreatic cell fate determination (11). Butyrate treatment was reported to improve $\beta$-cell proliferation, function and glucose homeostasis as well as to reduce $\beta$-cell apoptosis through HDAC inhibition and histone acetylation in diabetic 
rats (12). Li et al (13) observed that sodium butyrate-stimulated fibroblast growth factor 21 expression and enhanced fatty acid oxidation in the liver by inhibition of HDAC3. In addition, sodium butyrate has been demonstrated to improve systemic insulin sensitivity and increase energy expenditure in mice via upregulating mitochondrial function in skeletal muscle and brown fat through peroxisome proliferator-activated receptor $\gamma$ coactivator $1 \alpha(\mathrm{PGC}-1 \alpha)$ induction and elevation of adenosine monophosphate-activated protein kinase activity (8). However, the direct effect of butyrate on hepatic gluconeogenesis has remained to be elucidated.

Gluconeogenesis is an important pathological contributing factor in diabetic subjects and abnormally high during the progression of diabetes (14). In the liver, class IIa HDACs mediate glucogan-induced gluconeogenic gene expression through promoting deacetylation and activation of forkhead box O (FoxO) transcription factors. Hepatic knockdown of Class IIa HDACs in vivo resulted in lowered blood glucose in mice (15). Therefore, the present study investigated the direct effect of butyrate on gluconeogenesis in isolated mouse primary hepatocytes. Unexpectedly, butyrate significantly increased hepatic gluconeogenesis and the expression of gluconeogenic genes, which was different from the actions of other HDAC inhibitors.

\section{Materials and methods}

Materials. Hepatocyte medium was purchased from ScienCell (Carlsbad, CA, USA). Dulbecco's modified Eagle's medium (DMEM), Hank's balanced salt solution (HBSS), PBS and collagenase type IV (200 units/mg) were from Gibco (Thermo Fisher Scientific, Inc., Waltham, MA, USA). Sodium pyruvate, sodium L-lactate, dexamethasone, bovine serum albumin (BSA), 8-bromo-cyclic adenosine monophosphate (8-bromo-cAMP), acetate, propionate, sodium butyrate, Trichostatin A (TSA), CI994, Entinostat (MS-275), PCI-34051 and tubacin were obtained from Sigma-Aldrich (Merck KGaA, Darmstadt, Germany). All primers used for reverse-transcription quantitative polymerase chain reaction (RT-qPCR) were synthesized by Shanghai Biological Engineering Technology \& Services Co., Ltd. (Shanghai, China). Anti-cAMP response element-binding protein (CREB; cat no. 9197S) and anti-phosphorylated (p)-CREB (Ser133; cat no. 9198) as well as anti-mouse immunoglobulin (Ig)G (cat no. 14709) and anti-rabbit IgG conjugated with horseradish peroxidase (cat no. 7074) were from Cell Signaling Technology Inc. (Beverly, MA, USA). The antibody to GAPDH (cat no. sc25778) was purchased from Santa Cruz Biotechnology, Inc. (Danvers, MA, USA).

Isolation and culture of mouse primary hepatocytes. A total of 20 Male C57BL/6 mice (age, 6-8 weeks; weight, 16-18 g) were purchased from Shanghai Slack Experimental Center (Shanghai, China). Primary hepatocytes were isolated from C57BL/6 mice by a modified version of the collagenase method. In a biosafety cabinet, mouse livers were perfused with $10 \mathrm{ml}$ calcium-free HBSS through the portal vein under anesthesia with $10 \%$ chloral hydrate (Sigma-Aldrich; Merck, $\mathrm{KGaA})$. The liver was excised by careful dissection and transferred to a $10-\mathrm{cm}$ tissue culture dish. Mice were then sacrificed via cervical dislocation. The isolated liver was perfused with $0.05 \%$ collagenase IV (Gibco; Thermo Fisher Scientific, Inc.) dissolved in $20 \mathrm{ml}$ calcium-containing HBSS in a recirculating manner for $15 \mathrm{~min}$. Hepatocytes extracted from digested livers were filtered through a $100-\mu \mathrm{m}$ cell strainer, washed 3 times with PBS and re-suspended in hepatocyte medium containing $100 \mathrm{U} / \mathrm{ml}$ penicillin, $100 \mu \mathrm{g} / \mathrm{ml}$ streptomycin, $0.1 \%$ BSA and hepatocyte growth factor (all from ScienCell Research Laboratories, Inc., San Diego, CA, USA). The cells were seeded on 6-well or 24-well plates and incubated in a tissue culture incubator at $37^{\circ} \mathrm{C}\left(95 \%\right.$ air and $\left.5 \% \mathrm{CO}_{2}\right)$. After $24 \mathrm{~h}$, the cells had already attached to the plates and the medium was replaced with DMEM containing $5.5 \mathrm{mM}$ glucose and $0.25 \%$ BSA, followed by drug treatment. All mice used in the present study for isolation of hepatocytes were fed a normal diet under a regular schedule and were not fasted. All of the experimental procedures involving the use of animals were approved by the Animal Use and Care Committee of Shanghai Jiaotong University School of Medicine (Shanghai, China).

Cell culture and treatments. Mouse primary hepatocytes were cultured in DMEM supplemented with $0.25 \%$ BSA overnight at $37^{\circ} \mathrm{C}$ with $5 \% \mathrm{CO}_{2}$. To evaluate the effect of butyrate on hepatic gluconeogenesis, mouse primary hepatocytes were incubated with sodium butyrate for $8 \mathrm{~h}$ at various concentrations $(0,0.1,1,5$, or $10 \mathrm{mmol} / \mathrm{l})$. To investigate the effect of HDAC inhibitors on hepatic gluconeogenesis, cells were incubated in glucose-free DMEM containing gluconeogenic substrates $(10 \mathrm{mmol} / \mathrm{l}$ sodium lactate and $1 \mathrm{mmol} / 1$ sodium pyruvate), 8-bromo-cAMP (100 $\mu \mathrm{mol} / \mathrm{l})$ and with the following HDAC inhibitors: TSA (100 nmol/l), CI994 (10 $\mu \mathrm{mol} / 1)$, MS-275 (10 $\mu \mathrm{mol} / 1)$, PCI-34051 (10 $\mu \mathrm{mol} / 1)$, Tubacin $(10 \mu \mathrm{mol} / 1)$ or sodium butyrate (5 mmol/l). Following $8 \mathrm{~h}$ incubation at $37^{\circ} \mathrm{C}$, cell culture supernatants were collected for measuring the glucose content. Mouse primary hepatocytes were then treated with sodium butyrate $(5 \mathrm{mmol} / \mathrm{l})$ and TSA $(100 \mathrm{nmol} / \mathrm{l})$ in the presence of 8 -bromo-cAMP $(100 \mu \mathrm{mol} / \mathrm{l})$ for $8 \mathrm{~h}$ at $37^{\circ} \mathrm{C}$, the mRNA expression of gluconeogenic genes were detected using RT-qPCR. To compare the effects of three SCFAs on hepatic glucose production, mouse hepatocytes were treated with acetate $(5 \mathrm{mmol} / \mathrm{l})$, propionate $(5 \mathrm{mmol} / \mathrm{l})$ or sodium butyrate $(5 \mathrm{mmol} / \mathrm{l})$ for $8 \mathrm{~h}$ at $37^{\circ} \mathrm{C}$ in the presence of gluconeogenic substrates $(10 \mathrm{mmol} / 1$ sodium lactate and $1 \mathrm{mmol} / 1$ sodium pyruvate). To test whether SCFAs affect hepatic gluconeogenesis as substrates, mouse hepatocytes were incubated with one of three different SCFAs (acetate, propionate, or sodium butyrate; $5 \mathrm{mmol} / \mathrm{l}$ each) and $100 \mu \mathrm{M}$ 8-bromo-cAMP in the absence of gluconeogenic substrates (10 mmol/l sodium lactate and $1 \mathrm{mmol} / 1$ sodium pyruvate) for $8 \mathrm{~h}$ at $37^{\circ} \mathrm{C}$. To assess the phosphorylation levels of CREB, mouse hepatocytes were treated with butyrate $(5 \mathrm{mmol} / \mathrm{l})$ or 8-bromo-cAMP $(100 \mu \mathrm{mol} / \mathrm{l})$ for $1 \mathrm{~h}$ at $37^{\circ} \mathrm{C}$.

In vitro glucose production assay. Glucose production was assayed as described previously (16). In brief, hepatocytes were seeded into 24 -well plates at $2.5 \times 10^{5}$ cells/well. After $24 \mathrm{~h}$, these hepatocytes were pre-stimulated with DMEM containing $5.5 \mathrm{mM}$ glucose, $0.25 \%$ BSA and $100 \mathrm{nM}$ dexamethasone for $16 \mathrm{~h}$. Cells were then washed three times with 
Table I. Sequences of primers used for polymerase chain reaction.

\begin{tabular}{|c|c|c|c|}
\hline Gene & Sequence no. & Primer sequence $\left(5^{\prime}-3^{\prime}\right)$ & Product length (bp) \\
\hline PEPCK & NM_011044.2 & $\begin{array}{l}\text { F, GTGCTGGAGTGGATGTTCGG } \\
\text { R, CTGGCTGATTCTCTGTTTCAGG }\end{array}$ & 258 \\
\hline G6pase & NM_008061.4 & $\begin{array}{l}\text { F, ACTGTGGGCATCAATCTCCTC } \\
\text { R, CGGGACAGACAGACGTTCAGC }\end{array}$ & 344 \\
\hline FoxO1 & NM_019739.3 & $\begin{array}{l}\text { F, AAGAGCGTGCCCTACTTCAA } \\
\text { R, CTCCCTCTGGATTGAGCATC }\end{array}$ & 157 \\
\hline PGC-1 $\alpha$ & NM_008904.2 & $\begin{array}{l}\text { F, ATACCGCAAAGAGCACGAGAAG } \\
\text { R, CTCAAGAGCAGCGAAAGCGTCACAG }\end{array}$ & 253 \\
\hline $\mathrm{HNF} 4 \alpha$ & NM_008261.3 & $\begin{array}{l}\text { F, ATGCGACTCTCTAAAACCCTTG } \\
\text { R, ACCTTCAGATGGGGACGTGT }\end{array}$ & 135 \\
\hline$\beta$-actin & NM_007393.5 & $\begin{array}{l}\text { F, GGCTGTATTCCCCTCCATCG } \\
\text { R, CCAGTTGGTAACAATGCCATGT }\end{array}$ & 154 \\
\hline
\end{tabular}

FOX, forkhead box; PEPCK, phosphoenolpyruvate carboxykinase; G6pase, glucose 6-phosphatase; PGC1 $\alpha$, peroxisome proliferator-activated receptor $\gamma$ coactivator $1 \alpha$; HNF4 $\alpha$, hepatocyte nuclear factor $4 \alpha$; F, forward; R, reverse.

PBS and incubated in glucose production buffer (DMEM without glucose, serum or phenol red, and supplemented with $1 \mathrm{mM}$ sodium pyruvate and $10 \mathrm{mM}$ sodium lactate). After $8 \mathrm{~h}$, the cell culture supernatants were collected for measuring the glucose content using a glucose oxidase kit (Applygen Co., Beijing, China).

RNA extraction and RT- $q P C R$. Total RNA was extracted from mouse primary hepatocytes using TRIzol reagent (Invitrogen; Thermo Fisher Scientific, Inc.) according to the manufacturer's instructions. Complementary DNA was synthesized by RT using Random Primers (Promega Corp., Madison, WI, USA) according to the manufacturer's protocol. RT-qPCR was performed in a Roche LightCycler 480 system (Roche Diagnostics, Basel, Switzerland) using SYBR Premix EX Taq (Takara, Tokyo, Japan). The PCR conditions were as follows: Denaturation at $95^{\circ} \mathrm{C}$ for $10 \mathrm{sec}$, followed by 40 cycles of $95^{\circ} \mathrm{C}$ for $5 \mathrm{sec}$. The annealing temperature was then reduced to $60^{\circ} \mathrm{C}$ for $31 \mathrm{sec}$ with a final elongation step at $72^{\circ} \mathrm{C}$ for $300 \mathrm{sec}$. At the end of the amplification, a melting curve was generated in the temperature range of $60-95^{\circ} \mathrm{C}$. The sequences of primers used are listed in Table I. Relative gene expression levels were quantified based on the cycle threshold $(\mathrm{Cq})$ values and normalized to the reference gene $\beta$-actin. The relative gene expression was calculated using the $2^{-\Delta \Delta \mathrm{Cq}}$ method (17).

Western blot analysis. Primary hepatocytes were treated with radioimmunoprecipitation lysis buffer containing protease and phosphatase inhibitors (Merck $\mathrm{KGaA}$ ) and centrifuged at a speed of $6,000 \mathrm{x} \mathrm{g}$ for $10 \mathrm{~min}$ at $4^{\circ} \mathrm{C}$. The supernatant was then used to analyze the expression levels of specific proteins. Protein concentration was determined using a BCA protein assay kit (Thermo Fisher Scientific, Inc.). A total of $10 \mu \mathrm{g}$ protein was loaded per lane and separated in $15 \%$ SDS-PAGE. Samples were then transferred onto polyvinylidene fluoride membranes (ECL Advance; Cell Signaling Technology, Inc). Prior to western blot analysis, membranes were blocked with $5 \%$ skimmed milk for $2 \mathrm{~h}$ at $37^{\circ} \mathrm{C}$ and subsequently incubated at $4^{\circ} \mathrm{C}$ overnight with rabbit anti-mouse primary antibodies sourced from Cell Signaling Technology Inc. These included cAMP response element-binding protein (CREB; cat. no. 9197S; 1:1,000) and phosphorylated (p)-CREB (Ser133; cat. no. 9198; 1:1,000). GAPDH antibodies (cat. no. sc25778; 1:1,000) were purchased from Santa Cruz Biotechnology, Inc. Membranes were then incubated with mouse anti-rabbit horseradish peroxidase-labeled $\operatorname{IgG}$ secondary antibodies $(1: 2,000$; cat. no. 7074; Cell Signaling Technology Inc.,) overnight at $4^{\circ} \mathrm{C}$. The results were visualized using an immobilon ECL ultra western HRP substrate (cat. no. WBULS0100; Merck KGaA) and images were captured using an LAS-4000 Super CCD Remote Control Science Imaging System (Fuji, Tokyo, Japan).

Adenosine triphosphate (ATP) assay. The amount of ATP was measured by the luciferin-luciferase method according to the protocol of the ATP detection kit (cat. no. S0026; Beyotime Institute of Biotechnology, Inc., Haimen, China). Primary hepatocytes were seeded onto 24 -well plates at $2.5 \times 10^{5}$ cells/well. After $24 \mathrm{~h}$, these cells received the same dexamethasone pre-stimulation as described in the glucose production assay. The cells were then treated with propionate $(5 \mathrm{mmol} / \mathrm{l})$ and different concentrations of sodium butyrate $(0,0.1,1,5$ and $10 \mathrm{mmol} / \mathrm{l})$ for $1 \mathrm{~h}$ prior to lysis with lysis buffer $(200 \mu \mathrm{l} / \mathrm{well})$ from the ATP detection kit. After centrifugation at $12,000 \mathrm{x} \mathrm{g}$ for $5 \mathrm{~min}$ at $4^{\circ} \mathrm{C}$, the supernatant was transferred to a fresh tube for the ATP test. The luminescence of a 20- $\mu 1$ sample was assayed in a luminometer (Perkin Elmer, Inc., Waltham, MA, USA) together with $100 \mu \mathrm{l}$ ATP detection buffer from the ATP detection kit. The protein concentration was determined using a bicinchoninic acid protein assay kit (Thermo Fisher Scientific, Inc.). The concentration of ATP was normalized to that of protein in the same cell lysate.

Statistical analysis. All values are expressed as the mean \pm standard error of the mean from at least three 
independent experiments. Two group comparisons were performed using a Student's t-test. All statistical analyses were performed using SPSS 19.0 (IBM Corp., Armonk, NY, USA). $\mathrm{P}<0.05$ was considered to indicate a statistically significant difference.

\section{Results}

Sodium butyrate stimulates hepatic glucose production and gluconeogenic gene expression. To determine the effects of butyrate on hepatic gluconeogenesis, mouse primary hepatocytes were incubated with various concentrations of sodium butyrate for $8 \mathrm{~h}$. Unexpectedly, sodium butyrate stimulated hepatic glucose production in a dose-dependent manner, causing a significant increase at the concentration of $1 \mathrm{mM}(\mathrm{P}<0.01$; Fig. 1A). Under the same conditions, the expression of genes associated with hepatic gluconeogenesis, including phosphoenolpyruvate carboxykinase (PEPCK), glucose-6-phosphatase (G6Pase), FoxO1, PGC-1 $\alpha$ and hepatocyte nuclear factor $4 \alpha(\mathrm{HNF} 4 \alpha)$ was also increased. Consistent with the results on gluconeogenesis, sodium butyrate dose-dependently increased the mRNA expression of these gluconeogenic genes, with the maximum efficacy at the concentration of $10 \mathrm{mM}$ (Fig. 1B-F).

Effects of various HDAC inhibitors on hepatic gluconeogenesis. To investigate whether sodium butyrate-stimulated gluconeogenesis is involved in the inhibition of HDACs, the effects of other HDAC inhibitors, including TSA, CI994, MS-275, PCI-34051 and tubacin, on hepatic glucose production in mouse primary hepatocytes were detected. After mouse hepatocytes were incubated with $100 \mu \mathrm{M} 8$-bromo-cAMP for $8 \mathrm{~h}$, glucose production was markedly increased. In the presence of $100 \mathrm{nM}$ TSA, $10 \mu \mathrm{M}$ CI994 or $10 \mu \mathrm{M}$ PCI-34051, 8-bromo-cAMP-stimulated gluconeogenesis was significantly decreased. However, sodium butyrate treatment further enhanced glucose production induced by 8 -bromo-cAMP. The other two HDAC inhibitors had no significant effect (Fig. 2A). TSA is a potent inhibitor of class I and II HDAC (18). The present study further compared the effects of TSA and sodium butyrate on the expression of the five gluconeogenic genes. As expected, the expression of all of these gluconeogenic genes was strongly induced by 8 -bromo-cAMP. Contrary to the action of sodium butyrate, TSA suppressed cAMP-stimulated gluconeogenic gene expression (Fig. 2B-F). It is therefore unlikely that sodium butyrate promotes gluconeogenesis via inhibiting HDAC activity.

Effects of various SCFAs on hepatic glucose production and gluconeogenic gene expression. To investigate whether sodium butyrate stimulates gluconeogenesis as an energy substrate, the present study compared the effects of three SCFAs on hepatic glucose production. Mouse hepatocytes were treated with $5 \mathrm{mM}$ acetate, propionate or sodium butyrate for $8 \mathrm{~h}$ in the presence of gluconeogenic substrates (sodium lactate and sodium pyruvate). Similar to sodium butyrate, propionate also promoted hepatic glucose production (Fig. 3A). Sodium butyrate supplementation led to increases in the expression of all of the selected gluconeogenic genes. Propionate significantly triggered the mRNA expression of PEPCK, G6Pase,
FoxO1 and PGC-1 $\alpha$, but not that of HNF4 $\alpha$ (Fig. 3B-F). Although acetate had no significant effects on glucose production, it significantly increased the expression of FoxO1 and PGC-1 $\alpha$ (Fig. 3A, D and E).

Sodium butyrate stimulates hepatic glucose production as a substrate. To test whether sodium butyrate induces glucose production as a type of gluconeogenic substrate, mouse primary hepatocytes were incubated with one of three SCFAs in the absence of gluconeogenic substrates. Acetate, propionate and sodium butyrate all increased hepatic gluconeogenesis. Propionate had the most noticeable effect. In the presence of three SCFAs, addition of 8-bromo-cAMP further enhanced the glucose production (Fig. 4A). In agreement with the results on gluconeogenesis, 8-bromo-cAMP significantly enhanced butyrate and propionate-stimulated expression of the five gluconeogenic genes (Fig. 4B-F). Acetate supplementation only led to mild increases in PEPCK and PGC-1 $\alpha$ expression (Fig. 4B and E). It is well accepted that propionate is a substrate for hepatic glucose production (19). Therefore, it is reasonable to presume that butyrate also stimulates hepatic gluconeogenesis as a substrate.

Sodium butyrate activates CREB in mouse hepatocytes. It is well-known that cAMP/CREB is an important signaling pathway for hepatic gluconeogenesis $(20,21)$. The present study investigated whether the $\mathrm{CAMP} / \mathrm{CREB}$ signaling pathway is involved in butyrate-mediated induction of gluconeogenesis. As ATP is the substrate for cAMP production, the present study first detected the intracellular ATP concentration after mouse primary hepatocytes were incubated with various concentrations of sodium butyrate for $1 \mathrm{~h}$. In parallel with glucose production, sodium butyrate treatment resulted in the accumulation of intracellular ATP in a dose-dependent manner, exhibiting a significant effect at the concentration of $1 \mathrm{mM}(\mathrm{P}<0.05$; Fig. 5A). However, gluconeogenic substrates (sodium lactate and sodium pyruvate) as well as propionate did not alter the intracellular ATP concentration (Fig. 5B). Next, western blot analysis was performed to evaluate the phosphorylation state of CREB in butyrate-treated hepatocytes. Similar to 8-bromo-cAMP, sodium butyrate also stimulated the phosphorylation of CREB (Fig. 5C). Overall, these results indicated that butyrate activates CAMP/CREB signaling and stimulates the expression of hepatic gluconeogenic genes, at least in part via increasing the accumulation of intracellular ATP.

\section{Discussion}

The liver is the major site of gluconeogenesis from red blood cell-derived pyruvate and lactate and from amino acid precursors. Hepatic metabolism has a key role in the regulation of the energy status of the whole body. Increased glucose production through abnormally elevated hepatic gluconeogenesis is central to the manifestation of hyperglycaemia in type 2 diabetes (22). The present study demonstrated that butyrate promoted gluconeogenesis in mouse primary hepatocytes as a substrate and signaling molecule, which appears paradoxical to its beneficial effect on the whole-body energy metabolism in rodent animals. To the best of our knowledge, there is little 
A

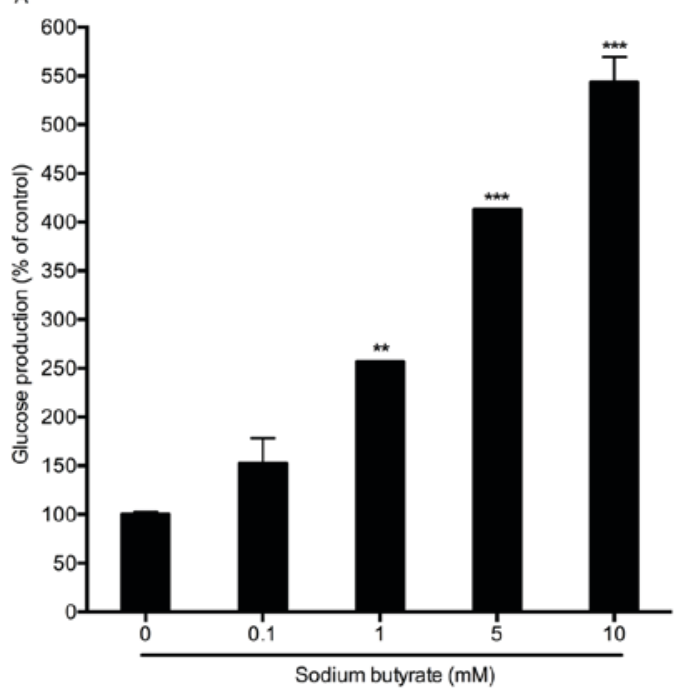

C

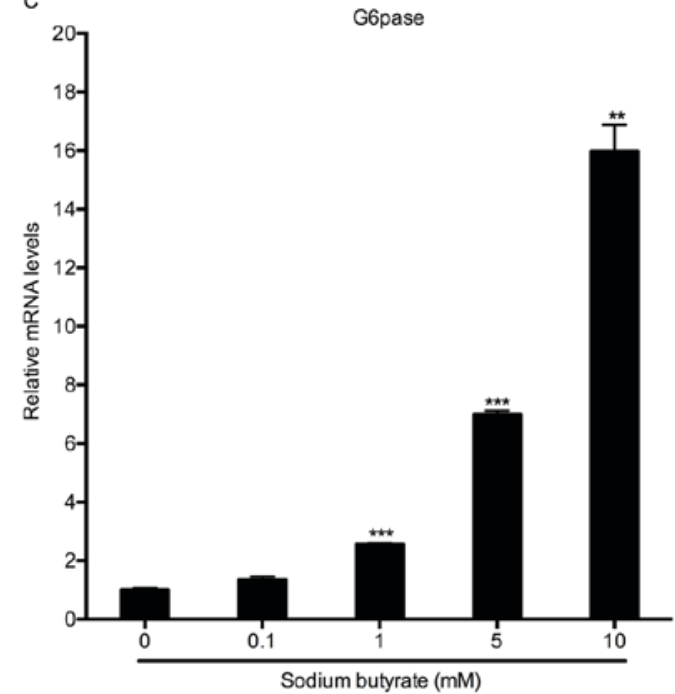

$\mathrm{E}$



B
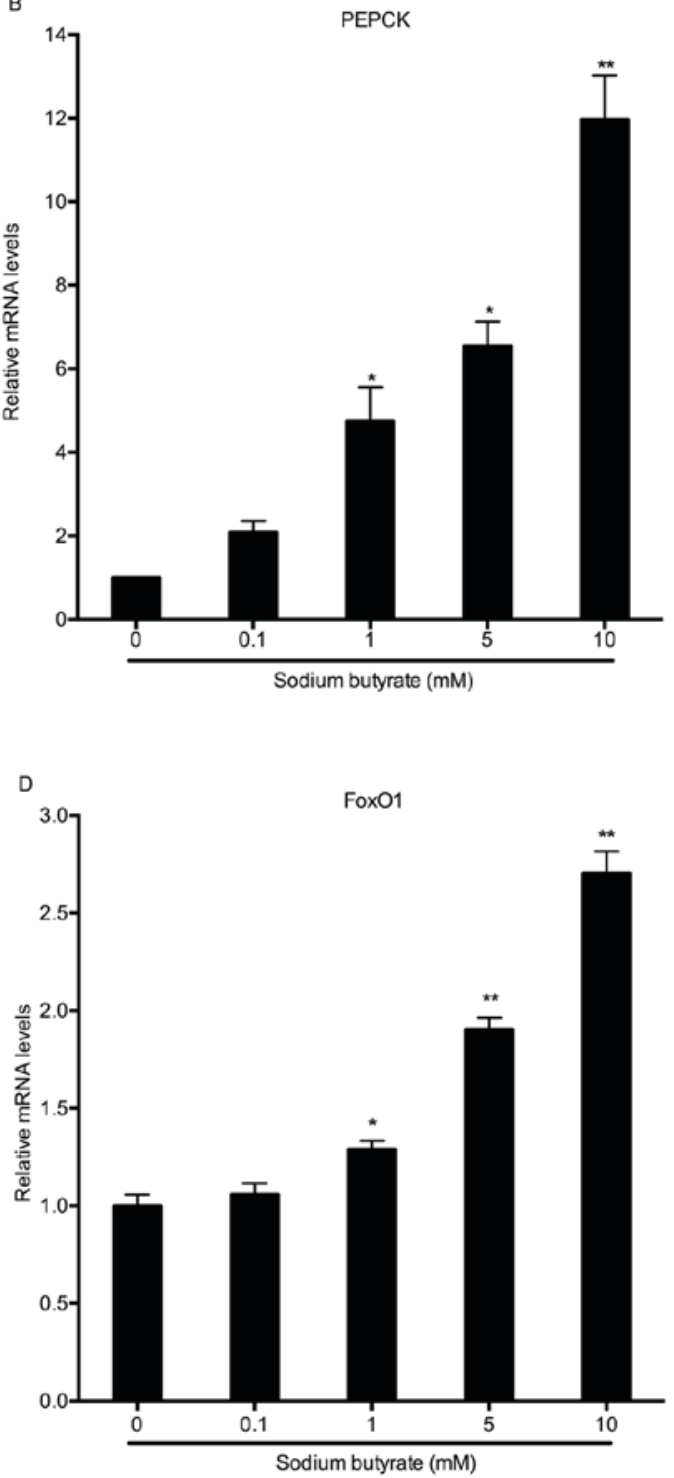

HNF4a

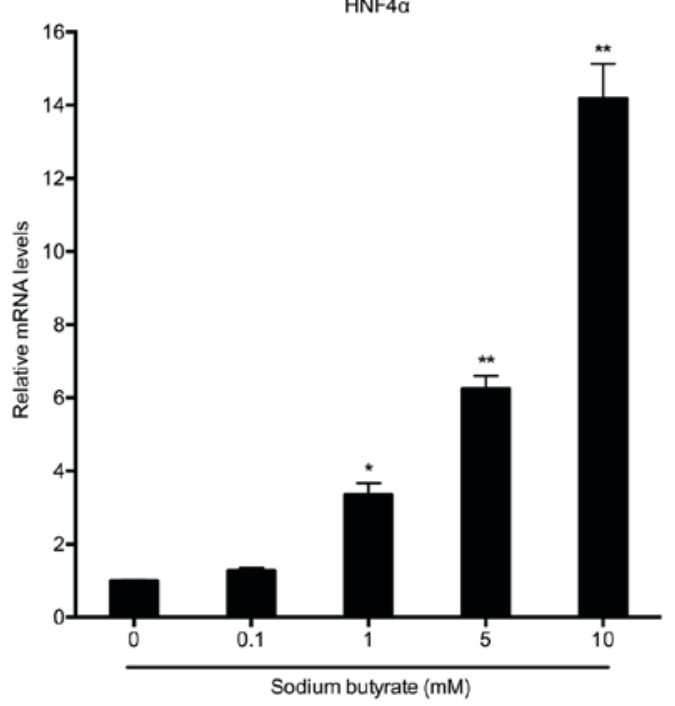

Figure 1. Sodium butyrate dose-dependently increases hepatic glucose production and gluconeogenic gene expression. (A) Mouse primary hepatocytes were incubated with the indicated concentrations of sodium butyrate for $8 \mathrm{~h}$. The cell culture supernatants were subsequently collected for measuring the glucose content. (B-F) The mRNA expression of gluconeogenic genes in mouse hepatocytes treated with sodium butyrate for $8 \mathrm{~h}$ was detected by reverse-transcription quantitative polymerase chain reaction analysis. Values are expressed as the mean \pm standard error of the mean of three separate experiments. " $\mathrm{P}<0.05$, ${ }^{* *} \mathrm{P}<0.01,{ }^{* * *} \mathrm{P}<0.001$ compared with control group. FOX, forkhead box; PEPCK, phosphoenolpyruvate carboxykinase; G6pase, glucose 6-phosphatase; PGC1 $\alpha$, peroxisome proliferator-activated receptor $\gamma$ coactivator $1 \alpha ; \mathrm{HNF} 4 \alpha$, hepatocyte nuclear factor $4 \alpha$. 
A

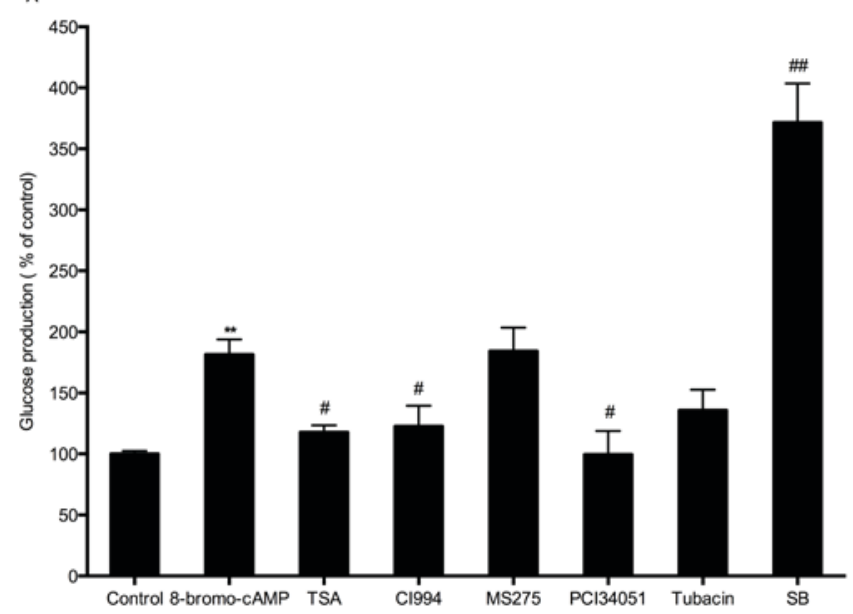

c

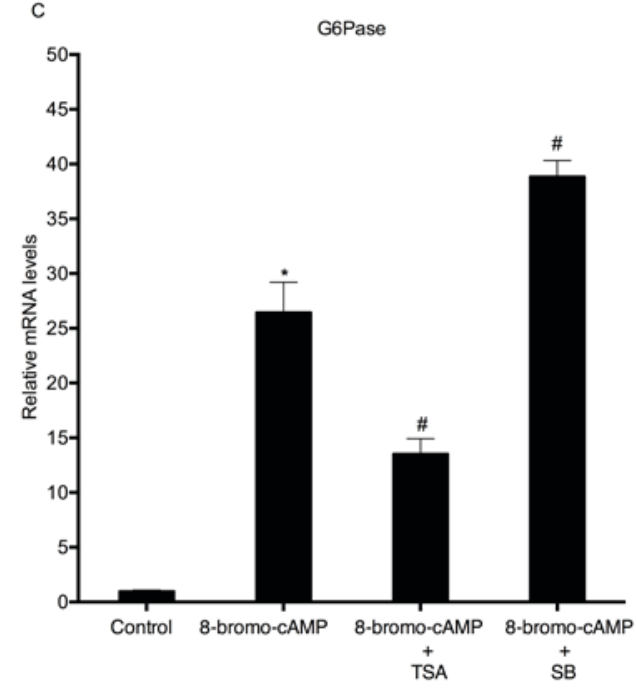

E

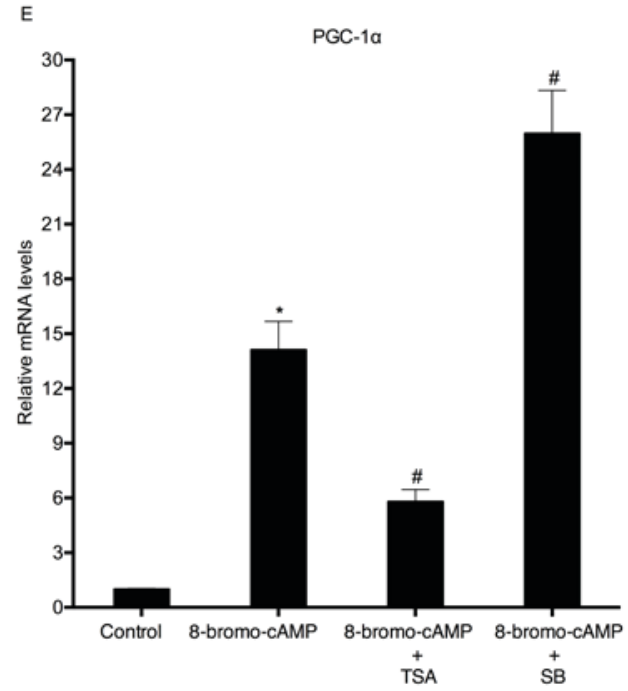

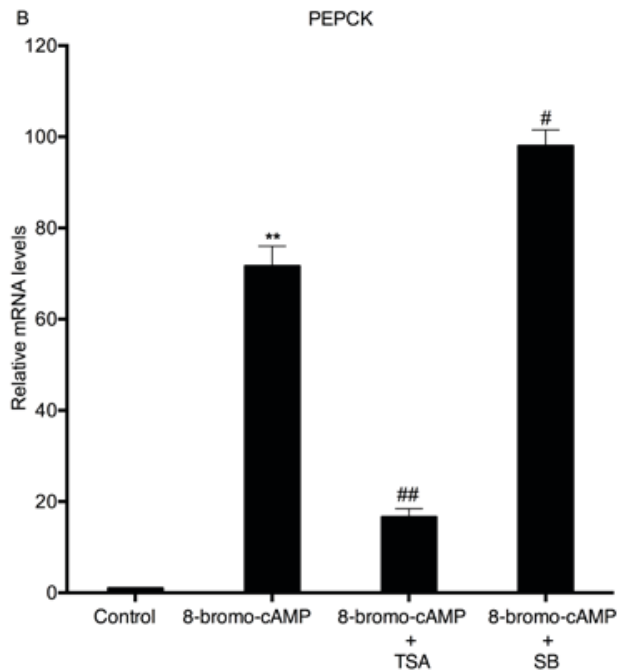

$\mathrm{D}$
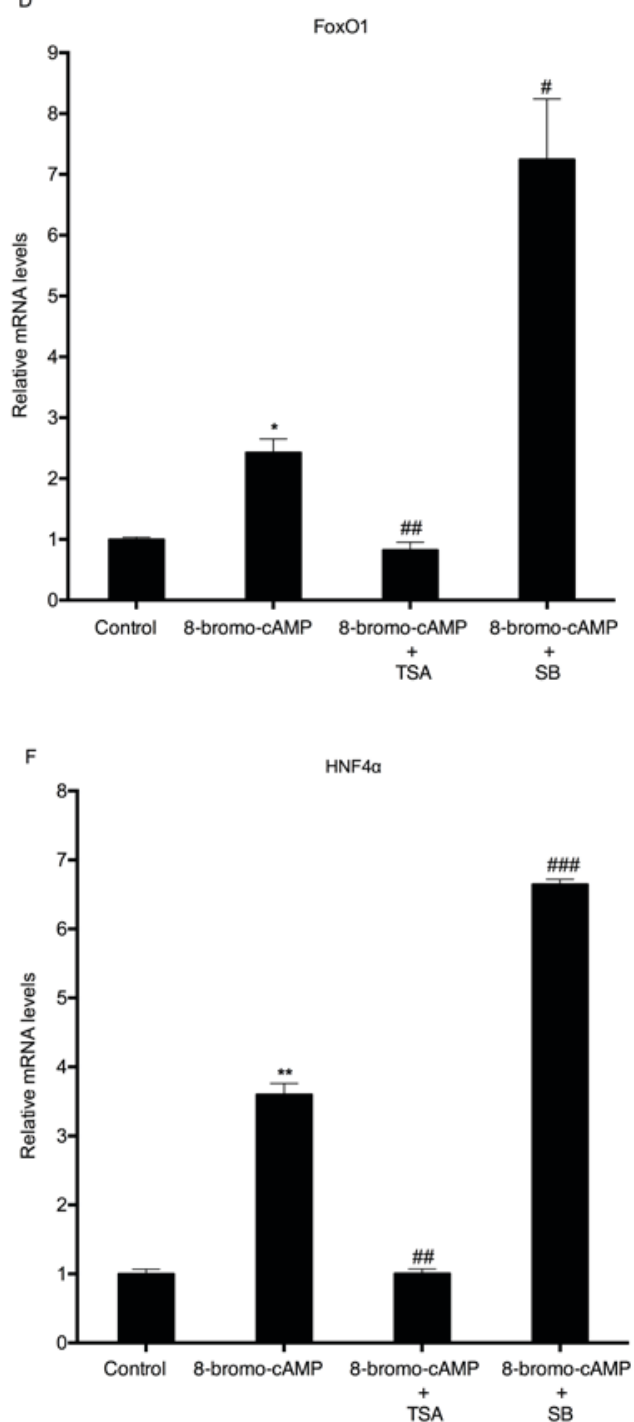

Figure 2. Effect of various HDAC inhibitors on hepatic glucose production. (A) Mouse hepatocytes were incubated with different HDAC inhibitors (100 nM TSA, $10 \mu \mathrm{M}$ CI994, $10 \mu \mathrm{M}$ MS-275, $10 \mu \mathrm{M}$ PCI-34051, $10 \mu \mathrm{M}$ Tubacin or $5 \mathrm{mM} \mathrm{SB}$ ) in glucose-free Dulbecco's modified Eagle's medium containing gluconeogenic substrates (10 mM sodium lactate and $1 \mathrm{mM}$ sodium pyruvate) and $100 \mu \mathrm{M} 8$-bromo-cAMP for $8 \mathrm{~h}$. The cell culture supernatants were collected for measuring the glucose content. (B-F) After mouse hepatocytes were treated with $5 \mathrm{mM}$ sodium butyrate and $100 \mathrm{nM}$ TSA in the presence of $100 \mu \mathrm{M} 8$-bromo-cAMP for $8 \mathrm{~h}$, the mRNA expressions of gluconeogenic genes were detected by reverse-transcription quantitative polymerase chain reaction analysis. Values are expressed as the mean \pm standard error of the mean of three separate experiments. ${ }^{*} \mathrm{P}<0.05$, ${ }^{* *} \mathrm{P}<0.01$ compared with control group; ${ }^{\#} \mathrm{P}<0.05,{ }^{\# \#} \mathrm{P}<0.01,{ }^{\# \# "} \mathrm{P}<0.001$ compared with 8-bromo-cAMP group. 8-bromo-cAMP, 8-bromo-cyclic adenosine monophosphate; TSA, Trichostatin A; MS-275, Entinostat; HDAC, histone deacetylase; SB, sodium butyrate; FOX, forkhead box; PEPCK, phosphoenolpyruvate carboxykinase; G6pase, glucose 6-phosphatase; PGCl $\alpha$, peroxisome proliferator-activated receptor $\gamma$ coactivator $1 \alpha$; HNF $4 \alpha$, hepatocyte nuclear factor $4 \alpha$. 
A

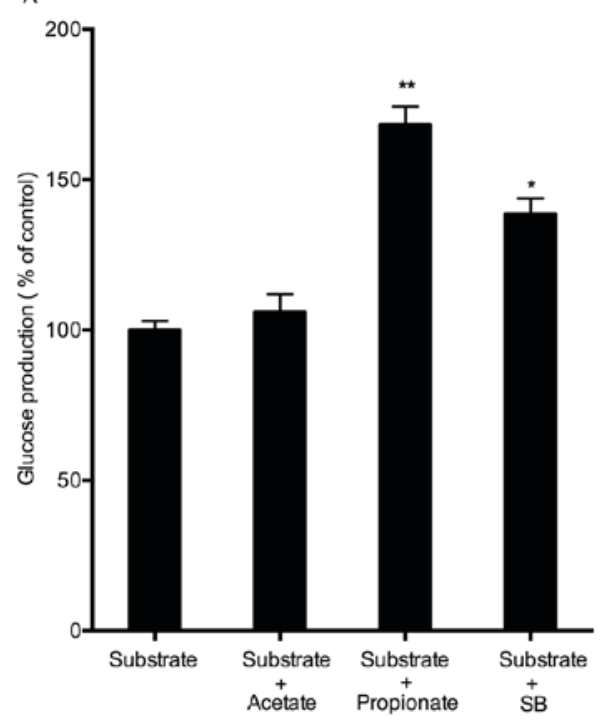

C

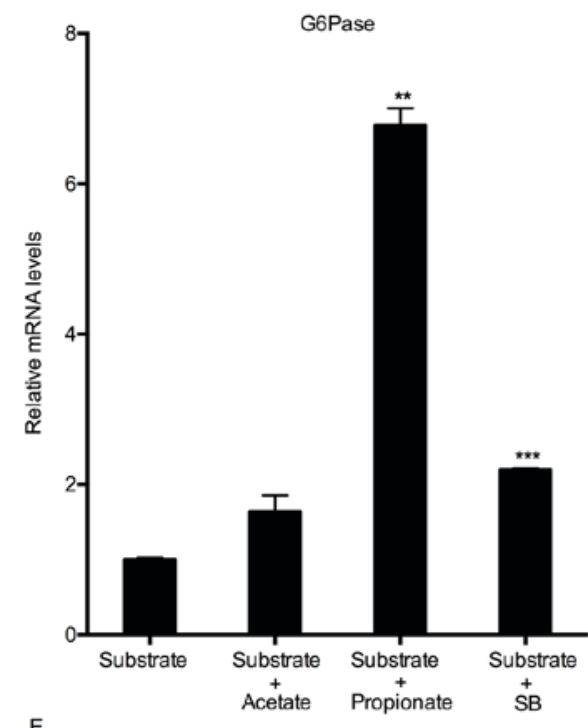

$\mathrm{E}$



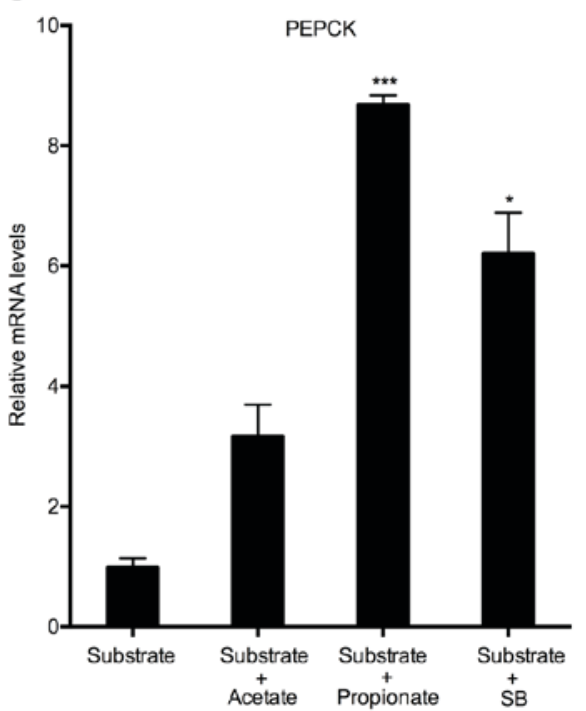

$\mathrm{D}$
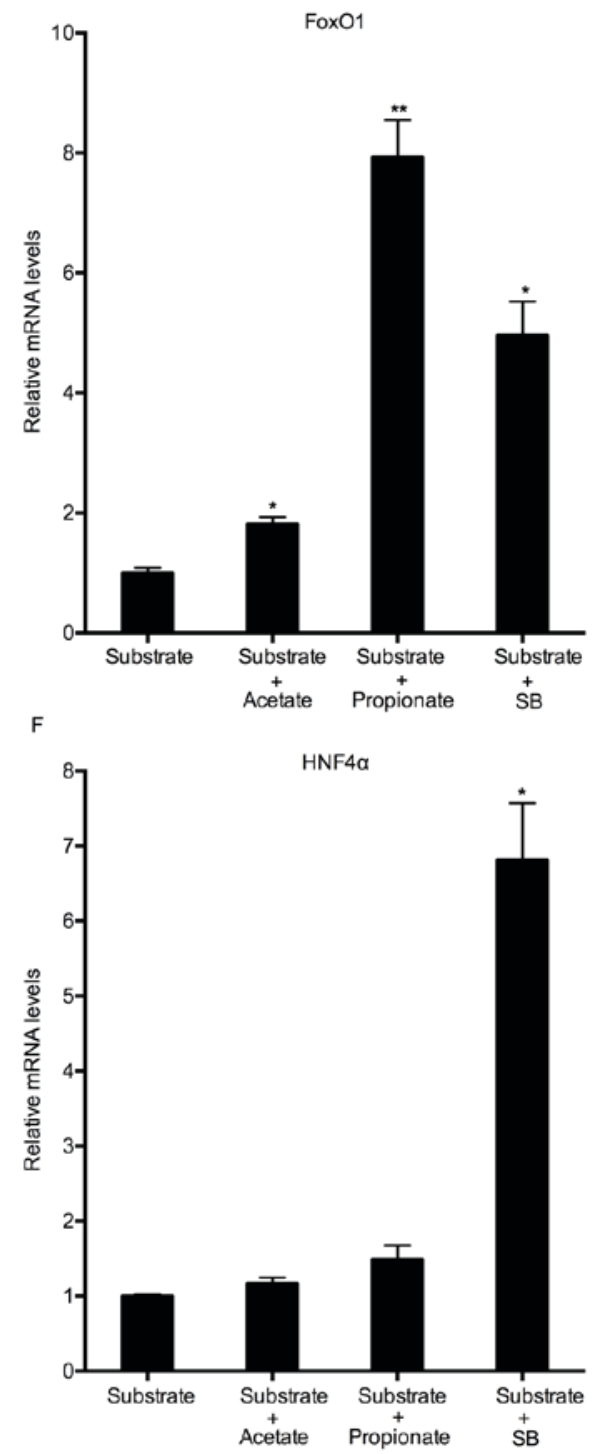

Figure 3. Effect of various short-chain fatty acids on hepatic glucose production and gluconeogenic gene expression. (A) Mouse hepatocytes were incubated with $5 \mathrm{mM}$ acetate, propionate or SB in glucose-free Dulbecco's modified Eagle's medium containing gluconeogenic substrates (10 mM sodium lactate and $1 \mathrm{mM}$ sodium pyruvate) for $8 \mathrm{~h}$. The cell culture supernatants were collected for measuring glucose content. (B-F) Under the same conditions, the mRNA expression of gluconeogenic genes was detected by reverse-transcription quantitative polymerase chain reaction analysis. Values are expressed as the mean \pm standard error of the mean of three separate experiments. ${ }^{*} \mathrm{P}<0.05,{ }^{* *} \mathrm{P}<0.01,{ }^{* * * *} \mathrm{P}<0.001$ compared with substrate group. SB, sodium butyrate; FOX, forkhead box; PEPCK, phosphoenolpyruvate carboxykinase; G6pase, glucose 6-phosphatase; PGCl $\alpha$, peroxisome proliferator-activated receptor $\gamma$ coactivator $1 \alpha ; H N F 4 \alpha$, hepatocyte nuclear factor $4 \alpha$. 

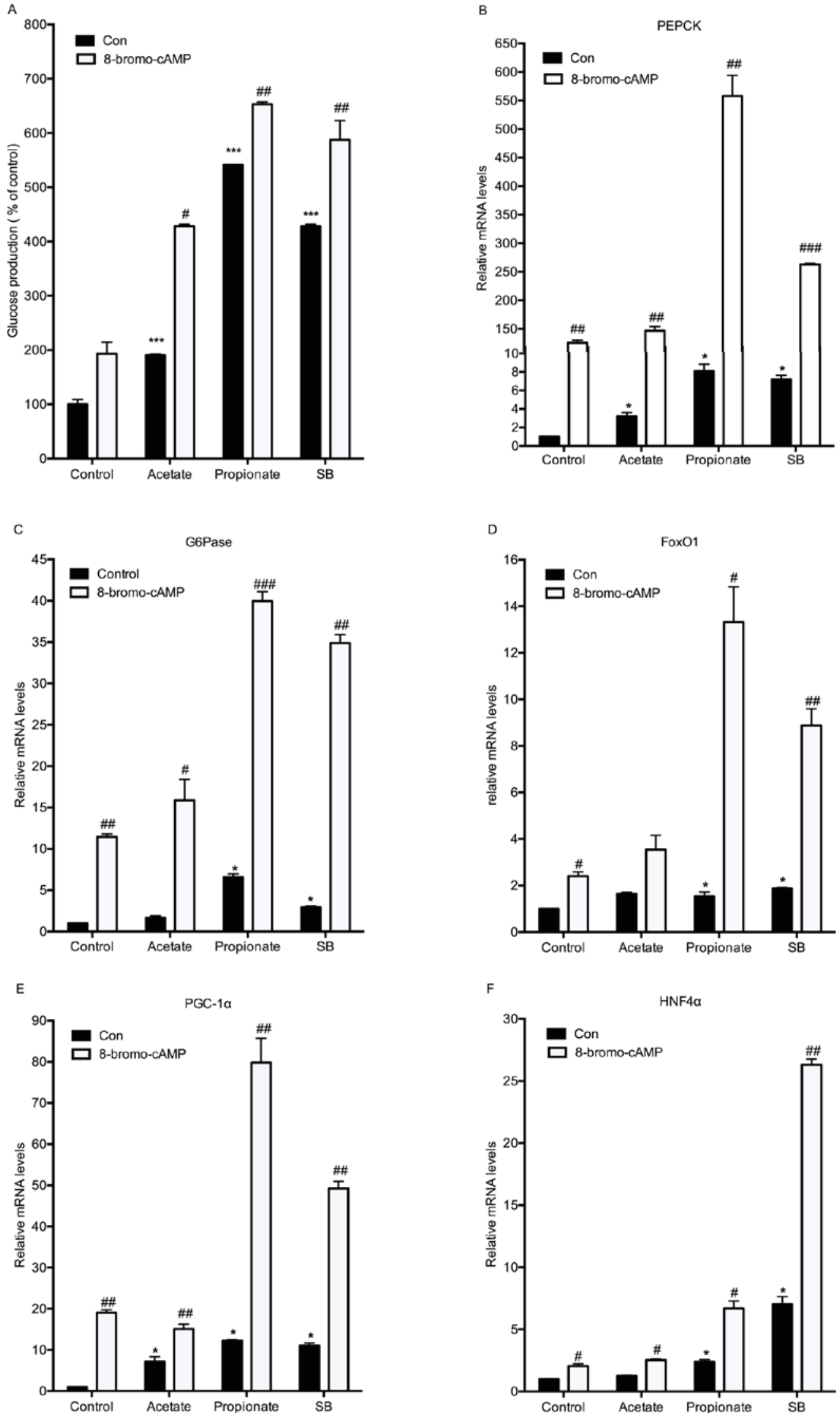

Figure 4. Sodium butyrate stimulates hepatic glucose production as a substrate. (A) Mouse hepatocytes were incubated with different short-chain fatty acids $(5 \mathrm{mM})$ and $100 \mu \mathrm{M}$ 8-bromo-cAMP in the absence of gluconeogenic substrates (sodium lactate and sodium pyruvate) for $8 \mathrm{~h}$. The cell culture supernatants were collected for measuring the glucose content. (B-F) Under the same conditions, the mRNA expression of gluconeogenic genes was detected by reverse-transcription quantitative polymerase chain reaction analysis. Values are expressed as the mean \pm standard error of the mean of three separate experiments. ${ }^{*} \mathrm{P}<0.05,{ }^{* * * *} \mathrm{P}<0.001$ compared with control group; ${ }^{\#} \mathrm{P}<0.05,{ }^{\# \#} \mathrm{P}<0.01,{ }^{\# \# \#} \mathrm{P}<0.001$ compared with CON group (without 8-bromo-cAMP). CON, control; 8-bromo-cAMP, 8-bromo-cyclic adenosine monophosphate; SB, sodium butyrate; FOX, forkhead box; PEPCK, phosphoenolpyruvate carboxykinase; G6pase, glucose 6-phosphatase; $\mathrm{PGCl} \alpha$, peroxisome proliferator-activated receptor $\gamma$ coactivator $1 \alpha$; HNF $4 \alpha$, hepatocyte nuclear factor $4 \alpha$. 

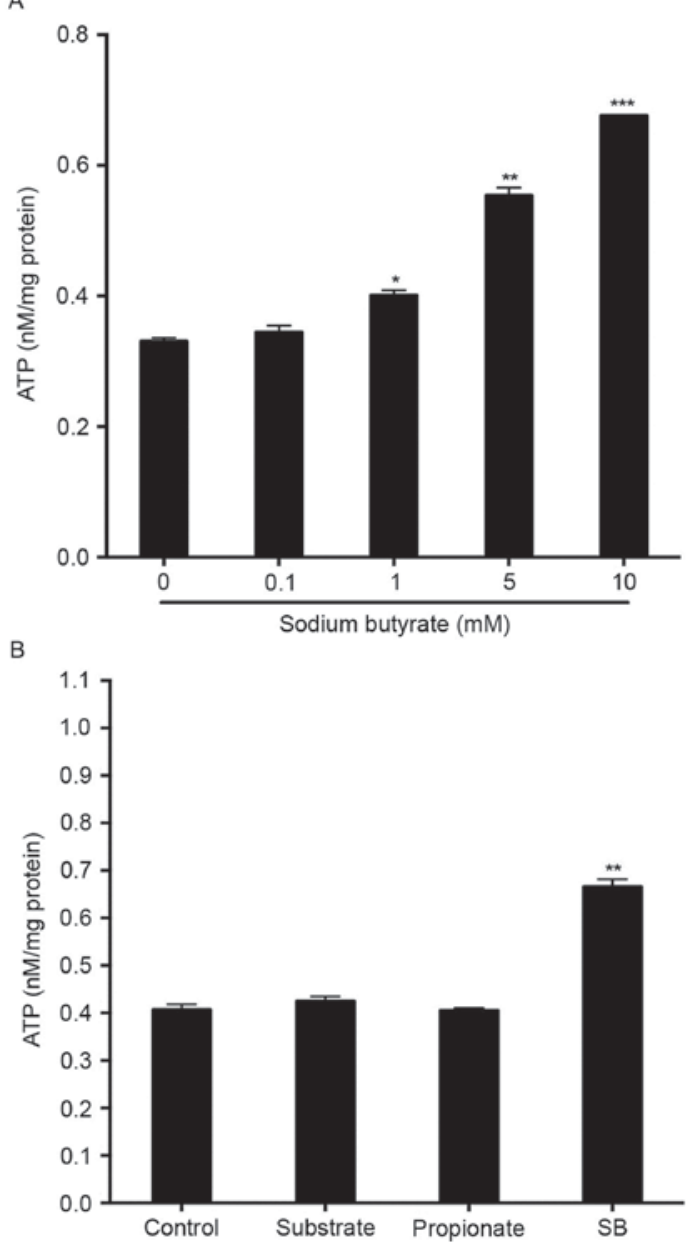

C

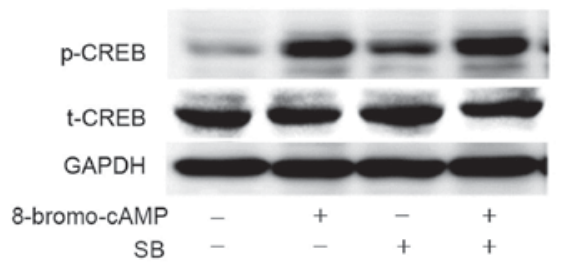

Figure 5. Effects of butyrate on intracellular ATP content and CREB phosphorylation. (A) Mouse hepatocytes were incubated with the indicated concentrations of sodium butyrate for $1 \mathrm{~h}$. Intracellular ATP concentration was measured by the luciferin-luciferase method. (B) Mouse hepatocytes were incubated with gluconeogenic substrates $(10 \mathrm{mM}$ sodium lactate and $1 \mathrm{mM}$ sodium pyruvate), $5 \mathrm{mM}$ proprionate, and $5 \mathrm{mM}$ butyrate for $1 \mathrm{~h}$. Intracellular ATP concentration was measured. (C) Mouse hepatocytes were treated with $5 \mathrm{mM}$ butyrate or $100 \mu \mathrm{M} 8$-bromo-cAMP for $60 \mathrm{~min}$ The phosphorylation level of CREB was detected by western blot analysis Values are expressed as the mean \pm standard error of the mean. ${ }^{*} \mathrm{P}<0.05$, ${ }^{* *} \mathrm{P}<0.01,{ }^{* * * *} \mathrm{P}<0.001$ compared with control group. ATP, adenosine triphosphate; $\mathrm{p} / \mathrm{t}$-CREB, phosphorylated/total cAMP response binding protein 8-bromo-cAMP, 8-bromo-cyclic adenosine monophosphate.

evidence demonstrating that butyrate directly improves blood glucose levels in human studies.

Gluconeogenesis is tightly controlled through the transcriptional regulation of PEPCK and G6Pase. Numerous transcription factors and co-activators, such as FoxO1, PGC- $1 \alpha$ and HNF4 $\alpha$, are involved in the induction of the two hepatic gluconeogenic genes (23). Class IIa HDAC, together with HDAC3, was reported to regulate hepatic gluconeogenesis via deacetylation of FoxO1 (15). Butyrate is a well-characterized
HDAC inhibitor. Similar to TSA, butyrate was reported to inhibit HDAC enzymes and increase the overall level of histone $\mathrm{H} 3$ acetylation (24). A previous study demonstrated that butyrate functioned as an HDAC inhibitor to stimulate the proliferation of colonocytes through changing their glucose metabolism (25). Therefore, the present study first investigated whether butyrate affected hepatic glucose production by acting as an HDAC inhibitor. Among six HDAC inhibitors, only sodium butyrate increased gluconeogenesis and TSA exerted the opposite effect. TSA is typically considered a broad-spectrum HDAC inhibitor. The present study further detected the expression of gluconeogenic genes in isolated mouse primary hepatocytes treated with sodium butyrate and TSA. In consistency with the results on gluconeogenesis, sodium butyrate enhanced 8-bromo-cAMP-stimulated hepatic gluconeogenic gene expression, while TSA had the opposite effect. These results indicated that butyrate stimulates gluconeogenesis and upregulates gluconeogenic gene expression independent of HDAC.

Besides being a broad-spectrum HDAC inhibitor, butyrate is an SCFA (6). In the present study, the effects of three SCFAs on hepatic glucose production and gluconeogenic gene expression were compared at the same concentration. Similar to sodium butyrate, propionate also stimulated gluconeogenesis and the expression of associated genes in mouse primary hepatocytes. Propionate has long been described as a hepatic gluconeogenic substrate (26). Infused propionate as a tracer was reported to markedly increase hepatic tricarboxylic acid metabolism and drive hepatic glucose production in a dose-dependent manner (19). Dietary supplementation with SCFAs, including propionate and butyrate, induced intestinal glucose production. Butyrate directly stimulated PEPCK and G6Pase expression in enterocytes in vitro (6). Therefore, it is likely that butyrate, as propionate, is also a gluconeogenic substrate. As expected, the present study demonstrated that sodium butyrate alone induced hepatic glucose production and gluconeogenic gene expression in mouse hepatocytes cultured with media void of other gluconeogenic substrates (sodium lactate and pyruvate).

cAMP has a pivotal role in the signaling pathways of hepatic gluconeogenesis (20). Since intracellular ATP is the substrate of cAMP production, the present study detected the intracellular ATP content in mouse hepatocytes incubated with butyrate and propionate. In agreement with the results of a previous study (27), propionate had no effect on the ATP concentration in the present study. However, butyrate dose-dependently induced the accumulation of intracellular ATP, demonstrating a similar increase to that during gluconeogenesis. Another mechanism underlying butyrate-stimulated gluconeogenesis except as a substrate has been reported; butyrate increased cAMP levels and protein kinase A activity in T-cells (28) and regulated the proliferation of porcine peripheral blood mononuclear cells in a cAMP-dependent manner (29). The present study revealed that butyrate stimulated the phosphorylation of CREB in mouse primary hepatocytes. These results suggested that butyrate stimulates gluconeogenesis via activating cAMP/CREB signaling.

In conclusion, the present study demonstrated that butyrate has a dual role in stimulating hepatic glucose production as a substrate and signaling molecule. cAMP/CREB signaling 
is involved in butyrate-induced hepatic gluconeogenic gene expression. Apparently, the beneficial effects of butyrate on other tissues counterbalances its action on hepatic gluconeogenesis, eventually improving the whole-body energy metabolism in rodent animals. However, additional studies should be performed before sodium butyrate is used in the treatment of type 2 diabetes mellitus in the clinic.

\section{Acknowledgements}

Not applicable.

\section{Funding}

This work was funded by grants from the National Natural Science Foundation of China (grant nos. 81270910, 81370876, 81471030, 81570693 and 30600294), the Natural Science Foundation of Shanghai (grant no. 15ZR1434000), and the Academic Leaders Training Program of Pudong Health Bureau of Shanghai (grant no. PWRd2011-01).

\section{Availability of data and materials}

The analyzed data sets generated during the present study are available from the corresponding author on reasonable request.

\section{Authors' contributions}

$\mathrm{XJ}$ and LZ designed the study. $\mathrm{XJ}$ and $\mathrm{FZ}$ conducted the experiments. YZ and $\mathrm{WX}$ analysed the data. XJ wrote the manuscript. LZ had primary responsibility for final content. All authors read and approved the final manuscript.

\section{Ethics approval and consent to participate}

All animal procedures were reviewed and approved by the Animal Use and Care Committee of Shanghai Jiao Tong University (Shanghai, China) and the China Experimental Animal Protection Association. All efforts were made to minimize the suffering of the experimental mice.

\section{Consent for publication}

Not applicable.

\section{Competing interests}

The authors declare that they have no competing interests.

\section{References}

1. Ning G: Decade in review-type 2 diabetes mellitus: At the centre of things. Nat Rev Endocrinol 11: 636-638, 2015.

2. Ley RE, Turnbaugh PJ, Klein S and Gordon JI: Microbial ecology: Human gut microbes associated with obesity. Nature 444: 1022-1023, 2006.

3. Papathanasopoulos A and Camilleri M: Dietary fiber supplements: Effects in obesity and metabolic syndrome and relationship to gastrointestinal functions. Gastroenterology 138 : 65-72.e1-e2, 2010.

4. Robertson MD, Currie JM, Morgan LM, Jewell DP and Frayn KN: Prior short-term consumption of resistant starch enhances postprandial insulin sensitivity in healthy subjects. Diabetologia 46: 659-665, 2003.
5. Robertson MD, Bickerton AS, Dennis AL, Vidal $\mathrm{H}$ and Frayn KN: Insulin-sensitizing effects of dietary resistant starch and effects on skeletal muscle and adipose tissue metabolism. Am J Clin Nutr 82: 559-567, 2005.

6. De Vadder F, Kovatcheva-Datchary P, Goncalves D, Vinera J, Zitoun C, Duchampt A, Bäckhed F and Mithieux G: Microbiota-generated metabolites promote metabolic benefits via gut-brain neural circuits. Cell 156: 84-96, 2014.

7. Robertson MD, Currie JM, Morgan LM, Jewell DP and Frayn KN: Prior short-term consumption of resistant starch enhances postprandial insulin sensitivity in healthy subjects. Diabetologia 46: 659-665, 2003.

8. Gao Z, Yin J, Zhang J, Ward RE, Martin RJ, Lefevre M, Cefalu WT and Ye J: Butyrate improves insulin sensitivity and increases energy expenditure in mice. Diabetes 58: 1509-1517, 2009.

9. Lin HV, Frassetto A, Kowalik EJ Jr, Nawrocki AR, Lu MM, Kosinski JR, Hubert JA, Szeto D, Yao X, Forrest G and Marsh DJ: Butyrate and propionate protectagainst diet-induced obesity and regulate gut hormones via free fatty acid receptor 3 -independent mechanisms. PLoS One 7: e35240, 2012.

10. Ellis L, Hammers H and Pili R: Targeting tumor angiogenesis with histone deacetylase inhibitors. Cancer Lett 280: 145-153, 2009.

11. Haumaitre $\mathrm{C}$, Lenoir $\mathrm{O}$ and Scharfmann R: Histone deacetylase inhibitors modify pancreatic cell fate determination and amplify endocrine progenitors. Mol Cell Biol 28: 6373-6383, 2008.

12. Khan S and Jena GB: Protective role of sodium butyrate, a HDAC inhibitor on beta-cell proliferation, function and glucose homeostasis through modulation of p38/ERK MAPK and apoptotic pathways: Study in juvenile diabetic rat. Chem Biol Interact 213: $1-12,2014$.

13. Li H, Gao Z, Zhang J, Ye X, Xu A, Ye J and Jia W: Sodium butyrate stimulates expression of fibroblast growth factor 21 in liver by inhibition of histone deacetylase 3. Diabetes 61: 797-806, 2012.

14. Hundal RS, Krssak M, Dufour S, Laurent D, Lebon V, Chandramouli V, Inzucchi SE, Schumann WC, Petersen KF, Landau BR and Shulman GI: Mechanism by which metformin reduces glucose production in type 2 diabetes. Diabetes 49: 2063-2069, 2000.

15. Mihaylova MM, Vasquez DS, Ravnskjaer K, Denechaud PD, Yu RT, Alvarez JG, Downes M, Evans RM, Montminy M and Shaw RJ: Class IIa histone deacetylases are hormone-activated regulators of FOXO and mammalian glucose homeostasis. Cell 145: 607-621, 2011.

16. Ip W, Shao W, Chiang YT and Jin T: The Wnt signaling pathway effector TCF7L2 is upregulated by insulin and represses hepatic gluconeogenesis. Am J Physiol Endocrinol Metab 303: E1166-E1176, 2012.

17. Livak KJ and Schmittgen TD: Analysis of relative gene expression data using real-time quantitative PCR and the 2(-Delta Delta C(T)) method. Methods 25: 402-408, 2001.

18. Taddei A, Roche D, Bickmore WA and Almouzni G: The effects of histone deacetylase inhibitors on heterochromatin: Implications for anticancer therapy? EMBO Rep 6: 520-524, 2005.

19. Perry RJ, Borders CB, Cline GW, Zhang XM, Alves TC, Petersen KF, Rothman DL, Kibbey RG and Shulman GI: Propionate increases hepatic pyruvate cycling, anaplerosis and alters mitochondrial metabolism. J Biol Chem 291: 12161-12170, 2016.

20. Altarejos JY and Montminy M: CREB and the CRTC co-activators: Sensors for hormonal and metabolic signals. Nat Rev Mol Cell Biol 12: 141-151, 2011.

21. Screaton RA, Conkright MD, Katoh Y, Best JL, Canettieri G, Jeffries S, Guzman E, Niessen S, Yates JR III, Takemori H, et al: The CREB coactivator TORC2 functions as a calcium- and cAMP-sensitive coincidence detector. Cell 119: 61-74, 2004.

22. Perriello G, Pampanelli S, Del Sindaco P, Lalli C, Ciofetta M, Volpi E, Santeusanio F, Brunetti P and Bolli GB: Evidence of increased systemic glucose production and gluconeogenesis in an early stage of NIDDM. Diabetes 46: 1010-1016, 1997.

23. Zhang W, Patil S, Chauhan B, Guo S, Powell DR, Le J, Klotsas A, Matika R, Xiao X, Franks R, et al: FoxO1 regulates multiple metabolic pathways in the liver: Effects on gluconeogenic, glycolytic, and lipogenic gene expression. J Biol Chem 281: 10105-10117, 2006.

24. Xiong H, Guo B, Gan Z, Song D, Lu Z, Yi H, Wu Y, Wang Y and Du H: Butyrate upregulates endogenous host defense peptides to enhance disease resistance in piglets via histone deacetylase inhibition. Sci Rep 6: 27070, 2016. 
25. Donohoe DR, Collins LB, Wali A, Bigler R, Sun W and Bultman SJ: The Warburg effect dictates the mechanism of butyrate-mediated histone acetylation and cell proliferation. Mol Cell 48: 612-626, 2012.

26. Anderson JW and Bridges SR: Short-chain fatty acid fermentation products of plant fiber affect glucose metabolism of isolated rat hepatocytes. Proc Soc Exp Biol Med 177: 372-376, 1984.

27. Massillon D, Arinze IJ, Xu C and Bone F: Regulation of glucose-6-phosphatase gene expression in cultured hepatocytes and H4IIE cells by short-chain fatty acids: Role of hepatic nuclear factor-4alpha. J Biol Chem 278: 40694-40701, 2003.

28. Diakos C, Prieschl EE, Saemann M, Novotny V, Bohmig G, Csonga R, Baumruker T and Zlabinger GJ: Novel mode of interference with nuclear factor of activated $\mathrm{T}$-cells regulation in T-cells by the bacterial metabolite n-butyrate. J Biol Chem 277: 24243-24251, 2002.
29. Weber TE and Kerr BJ: Butyrate differentially regulates cytokines and proliferation in porcine peripheral blood mononuclear cells. Vet Immunol Immunopathol 113: 139-147, 2006.

This work is licensed under a Creative Commons Attribution-NonCommercial-NoDerivatives 4.0 International (CC BY-NC-ND 4.0) License. 